\title{
Options for decarbonization of future transport
}

Dr. Andreas Kolbeck, Co-authors: Dr. Wolfgang Warnecke, Dr. Christoph Balzer, Dr. Karsten Wilbrand, all Shell Global Solutions (Deutschland) $\mathrm{GmbH}$

Die Unterlagen wurden nicht zur Veröffentlichung freigegeben. Wir bitten um Verständnis. 